\title{
DOS NUEVOS REGLAMENTOS DE LA UNIÓN EUROPEA PARA FACILITAR LA LIBRE CIRCULACIÓN (DE TRABAJADORES Y DE PERSONAS)
}

\author{
Joaquín GARCía MurCia \\ Departamento de Derecho del Trabajo \\ y de la Seguridad Social \\ Facultad de Derecho de la UCM \\ jgmurcia@der.ucm.es
}

\section{ALGUNAS INDICACIONES BÁSICAS SOBRE LA LIBRE CIRCULACIÓN (DE TRABAJADORES Y DE PERSONAS)}

La libertad de circulación ha sido sin duda alguna uno de los principales soportes en la construcción de la actual Unión Europea. Fue concebida en un primer momento como un instrumento para la movilidad de los trabajadores en el espacio europeo (como aún se sigue consignando en el título IV del Tratado de Funcionamiento de la Unión Europea), y pasó a ser desde hace algunas décadas un derecho de ciudadanía (como quedó reflejado en su momento en el art. 3 del Tratado de la Unión). Ambas facetas, la profesional y la personal, forman parte ya de la tabla de derechos fundamentales de la Unión Europea (según puede comprobarse en los arts. 15 y 45 de la Carta del año 2000), y ambas cuentan, como no podía ser de otro modo, con la correspondiente recepción dentro del Derecho comunitario de carácter derivado. Tras una interesante sucesión normativa, la primera de esas dimensiones de la libre circulación se regula principalmente en el Reglamento 492/2011, mientras que la segunda, de más moderna configuración y regulación, tiene su tratamiento básico en la Directiva 2004/38. Como es natural, son muchos los puntos de contacto entre uno y otro segmento normativo, pues uno y otro tratan, a fin de cuentas, de facilitar los movimientos de personas en el territorio de la Unión Europea y de proporcionar la debida protección a quienes ejercitan esos derechos de movilidad; ambos tratan, asimismo, de evitar situaciones de peligro para el orden público o perjuicios para la seguridad y salud públicas, si los queremos ver desde esta otra perspectiva. 
Pero tampoco faltan las diferencias en el tratamiento de una y otra faceta de la libre circulación, entre otras razones porque la migración por razones de trabajo no puede equipararse por completo al mero cambio de residencia decidido por una persona, ni por sus necesidades logísticas, ni por el escenario (vital, familiar e incluso social) al que da o puede dar lugar. La persona que circula por motivos profesionales requiere sobre todo oportunidades en la búsqueda de empleo, protección en sus condiciones de trabajo y asistencia en las condiciones ligadas al trabajo (como es el caso de la seguridad social), mientras que la persona que se mueve de un país a otro por motivos distintos del trabajo (aunque no se descarte por completo la búsqueda de empleo) necesita antes que nada facilidades para su adecuada reubicación en un nuevo contexto ciudadano (es decir, sobre todo, facilidades de naturaleza administrativa o burocrática). No es exactamente igual, por otra parte, migrar como trabajador, lo que presupone la posesión —o la posibilidad razonable de posesión - de una determinada fuente de ingresos, que cambiar de residencia al margen de esa circunstancia, lo cual puede aconsejar, como la experiencia ha venido demostrando, cierta prevención o cierto cuidado respecto de los medios de vida o las expectativas de ingresos de quien se traslada de un país a otro. Por lo demás, el hecho de que la regulación comunitaria de la libertad de circulación de los trabajadores se encuentre en un reglamento y de que, en cambio, la regulación de la libertad de circulación de personas se recoja en una directiva, también puede servir de advertencia acerca del distinto grado de articulación entre intervención supranacional e intervención estatal que el Derecho de la Unión Europea reserva para cada uno de esos supuestos (aun cuando tampoco parezca existir, ciertamente, un criterio absolutamente determinante para la opción entre una y otra categoría normativa dentro de este terreno, como da a entender, por ejemplo, el hecho relativamente reciente de que el refuerzo de la libre circulación de trabajadores se haya articulado a través de una norma de armonización, la Directiva 2014/54, y no de una norma de aplicación directa).

De cualquier manera, y junto a las mencionadas normas básicas, tanto la libre circulación de trabajadores como la libre circulación de personas han ido generando o reclamando el auxilio de otras muchas normas de carácter complementario, con las que las instituciones comunitarias han tratado de dar respuesta a sus efectos secundarios o sus problemas colaterales. Alrededor de la libre circulación de trabajadores nació desde muy temprano todo un conglomerado normativo dedicado específicamente a la coordinación de los sistemas nacionales de seguridad social, y a su abri- 
go han ido surgiendo también, con el transcurso de los años, otras muchas intervenciones normativas a escala europea, algunas de ellas pertenecientes por completo a esta parcela de la libre circulación (como la ya citada Directiva 2014/54, que ha procedido al refuerzo de la posición jurídica y los derechos de tutela jurisdiccional de los trabajadores migrantes), y algunas otras insertas en una regulación de contornos más amplios o de proyección más generalizada desde el punto de vista material y funcional (como las reglas dedicadas a determinar la ley aplicable y el foro competente en los casos de concurrencia de dos o más sistemas nacionales, o las reglas dedicadas al reconocimiento y la convalidación de títulos y diplomas profesionales). Por su parte, de la libre circulación de personas también han venido emanando algunas disposiciones normativas de carácter complementario, que en este caso se dedican principalmente a garantizar o reforzar la cooperación administrativa entre las pertinentes instituciones nacionales (como fue el caso del Reglamento 1024/2012 sobre intercambio de información, ahora parcialmente modificado).

En el año 2016 han aparecido precisamente dos importantes normas sobre libre circulación pertenecientes a este circuito complementario, en ambos casos para modernizar reglas precedentes o para precisar algunos de sus extremos. No son, en consecuencia, intervenciones normativas absolutamente innovadoras, aunque sí muestran un nítido propósito de acondicionamiento o actualización de la normativa preexistente. La primera de esas normas, por orden cronológico, es el Reglamento UE 2016/589, de 13 de abril, relativo a la red europea de servicios de empleo (EURES), que, como podrá comprenderse fácilmente, se ocupa sobre todo de los aspectos laborales o profesionales de la libre circulación. La segunda de ellas es el Reglamento UE 2016/1191, de 6 de julio, que busca ante todo la simplificación de las exigencias documentales que suele conllevar el movimiento de personas de unos países a otros, y que se inscribe de manera más directa en el principio de libre circulación de personas o ciudadanos. La primera norma ha sido aprovechada también para modificar los Reglamentos UE 492/2011 (regulación básica en materia de libre circulación de trabajadores, como sabemos) y 1296/2013 (impulsor del Programa para el Empleo y la Innovación Social dentro de la Estrategia Europa 2020), mientras que la segunda ha incidido sobre todo en los instrumentos de cooperación comprendidos en el Reglamento UE 1024/2012, que se encargó de modernizar, a su vez, el Sistema de Información del Mercado Interior que se había puesto en marcha mediante diversas intervenciones normativas anteriores de la Unión Europea. 


\section{LA RED EURES DE INFORMACIÓN Y COMPENSACIÓN DE OFERTAS Y DEMANDAS DE EMPLEO}

El Reglamento 2016/589 conecta de manera muy directa con dos previsiones básicas de la libre circulación de trabajadores. Por un lado, con el art. 46 TFUE, donde se exige a las instituciones europeas el establecimiento de «mecanismos adecuados para poner en relación las ofertas y las demandas de empleo y facilitar su equilibrio en condiciones tales que no se ponga en grave peligro el nivel de vida y de empleo en las diversas regiones e industrias». Por otro lado, con las normas de Derecho derivado reguladoras de la libre circulación de trabajadores, que con carácter general llamaban a la colaboración entre los Estados miembros y la Comisión para hacer efectiva la movilidad de la mano de obra y que, de forma especial, exigían a los servicios centrales de empleo de los Estados miembros que cooperaran «estrechamente entre sí y con la Comisión, con miras a conseguir una acción común en el campo de la compensación de las ofertas y demandas de empleo en la Unión y la colocación de trabajadores que de ella resulte» (como literalmente decía el art. 11 del Reglamento 492/2011, ahora sustituido). El Reglamento 2016/589, de ese modo, viene a renovar, reforzar y «restablecer» (según sus propias palabras) la red de compensación de ofertas y demandas de empleo que ya venía funcionando en el espacio comunitario desde el año 1994, y que desde entonces es conocida como «Red EURES».

En el momento de aprobación de esta nueva norma, la red EURES estaba regulada básicamente por el art. 11 del Reglamento 492/2011 (que, repetimos, ha sido expresamente suprimido por el Reglamento 2016/589) y por la Decisión 2012/733/UE (que también ha sido derogada, en este caso por la Decisión 2016/716/UE). Conviene tener en cuenta, por otra parte, que la cooperación de los servicios públicos de empleo en el ámbito comunitario había sido impulsada asimismo por la Decisión 572/2014/UE, que a tal efecto, y dentro de la Estrategia Europa 2020, había creado una red específica para la conexión de dichos servicios para el periodo comprendido entre 17 de junio de 2014 y 31 de diciembre de 2020 (conocida como «Red SPE», esto es, red de los servicios públicos de empleo). El Reglamento 2016/589 deja en funcionamiento esta red paralela de cooperación administrativa (con la que en todo caso abre las oportunas vías de colaboración), y, además, traza líneas de contacto con otras muchas redes de empleo (como la red Equinet para la igualdad, el portal «Tu Europa» 
o el portal Europeo de la Juventud y Solvit) y con numerosas organizaciones o instituciones dedicadas, o bien a la promoción y el fomento del empleo (organizaciones de cooperación transfronteriza, de reconocimiento de las cualificaciones profesionales o de apoyo de la igualdad de trato en el empleo), o bien a la protección de quienes migran de unos países a otros por razones de empleo (como las organizaciones de apoyo en el terreno de la coordinación de los sistemas nacionales en materia de seguridad social). El Reglamento 2016/589 quiere erigirse, así, en el eje normativo de todo este entramado de instrumentos o medios de coordinación de los sistemas nacionales.

La nueva reglamentación de la red EURES parte de la necesidad de revisar el marco normativo precedente para tomar conciencia de las nuevas dimensiones y las nuevas necesidades del mercado de trabajo. Literalmente, y de acuerdo con su preámbulo, trata de reflejar «las nuevas pautas de movilidad, la mayor exigencia de una movilidad equitativa, los cambios en la tecnología de intercambio de datos sobre ofertas de empleo, el uso de una variedad de canales de contratación por los trabajadores y los empresarios, y un papel cada vez más importante de otros agentes del mercado laboral, junto con los servicios públicos de empleo (SPE), en la prestación de servicios de contratación». También consideran sus promotores que la «creciente interdependencia entre los mercados de trabajo requiere una mayor colaboración entre los servicios de empleo, incluidos los de las regiones transfronterizas», que es conveniente establecer un marco de mayor cooperación entre la Comisión y los Estados miembros, y que debe abordarse el mercado de trabajo con una visión amplia, partiendo de la noción flexible de «trabajador» defendida por el Tribunal de Justicia, lo que aconseja prestar atención no sólo a las situaciones de trabajo estricto, sino también a las de aprendizaje y prácticas (en línea, por lo demás, con la Recomendación del Consejo de 10 de marzo de 2014, que proporciona «un marco de calidad para los periodos de prácticas»). Se requiere, a la postre, mayor extensión, coherencia y flexibilidad en el funcionamiento de la red de intercambios y compensaciones de ofertas y demandas de empleo, una red que se quiere abrir también a la participación de los agentes sociales.

El Reglamento 2016/589 — que, con alguna excepción, entra en vigor al vigésimo día de su publicación oficial, que tuvo lugar en el DOUE de 22 de abril de 2016 — procede en esencia a una profunda reorganización de la red EURES, tratando de superar las deficiencias advertidas en la configuración que para dicha red se había previsto en las normas inmediatamente precedentes, esto es, en el Reglamento 492/2011 y en la Decisión 2012/733. Dis- 
pone el Reglamento 2016/589, en primer término, que los servicios comprendidos en la red EURES quedan «a disposición de todos los trabajadores y empresarios de la Unión», y que dichos servicios «respetarán el principio de igualdad de trato» y habrán de garantizar, en especial, «la accesibilidad de las personas con discapacidad» (art. 4). A ello se añade que la red EURES habrá de contribuir a la consecución de unos cuantos objetivos básicos (art. 6): por lo pronto, al ejercicio de los derechos de libre circulación reconocidos en la normativa comunitaria, pero también a la «aplicación de la estrategia coordinada para el empleo» y para la «promoción de una mano de obra capacitada, formada y adaptable» (de conformidad con el art. 145 TFUE), a la mejora del «funcionamiento, la cohesión e integración de los mercados de trabajo en la Unión, también a nivel transfronterizo», al fomento de la movilidad geográfica y profesional voluntaria dentro de la Unión, y, en fin, al apoyo de las «transiciones al mercado de trabajo» (en concordancia con los objetivos sociales y de empleo del art. 3 TUE).

Con el Reglamento 2016/589, la red EURES queda compuesta de una amplia serie de organismos y medios de acción (art. 7). En primer término se organiza alrededor de una Oficina Europea de Coordinación (establecida dentro de la Comisión y responsable de asistir a la red EURES en el desempeño de sus actividades), y de unas Oficinas Nacionales de Coordinación $(\mathrm{ONC})$, que son designadas por los Estados miembros, son responsables de la aplicación de estas reglas en el interior de los mismos, pueden coincidir con los correspondientes servicios públicos de empleo, y se relacionan con aquella Oficina Europea a través de un Grupo de Coordinación. Por otra parte, la red consta de una serie de «miembros», categoría que corresponde no sólo a los servicios públicos de empleo designados por los Estados miembros, sino también a otras instancias u organizaciones de apoyo a trabajadores y empresarios, siempre que sean admitidas y designadas formalmente por los Estados miembros con arreglo a los procedimientos que el propio Reglamento 2016/589 establece. También cabe la designación de «socios» de la red EURES, calificación que puede otorgarse a las organizaciones que acreditan determinadas condiciones (todo ello conforme a lo dispuesto en el art. 11 del Reglamento y en sus reglas conexas, algunas de ellas destinadas a mantener la categoría de «miembro» o «socio» a organizaciones que ya la habían adquirido con anterioridad). Las organizaciones de trabajadores o empresarios pueden formar parte de la red EURES como miembros o como socios. La cualidad de miembro o socio de EURES aporta naturalmente determinadas ventajas, pero también entraña exigencias y obligaciones: todos esos sujetos deben contribuir al buen funcionamiento de la 
red mediante la aportación de ofertas o demandas de empleo o la prestación de servicios de apoyo a trabajadores y empresarios, entre otras tareas que les pueden requerir las instituciones públicas competentes.

Para que se cumpla la principal función de esta red («la puesta en correspondencia de las ofertas y las demandas de empleo»), cada Estado miembro debe publicar en el portal EURES todas las ofertas y demandas de empleo que publiquen en sus servicios públicos de empleo, bien es cierto que con la posibilidad de hacer salvedades por motivos justificados e incluso de excluir determinadas ofertas de empleo por razones que pueden comprenderse sin mayor dificultad: las ofertas que, debido a su naturaleza o a las normas nacionales, sólo estén disponibles para los ciudadanos de un país determinado; las que estén relacionadas con categorías de periodos de aprendizaje o de prácticas que, teniendo principalmente un componente de formación, formen parte de los sistemas educativos nacionales o cuenten con financiación pública como parte de las políticas activas del mercado de trabajo de los Estados miembros, y, en general, las ofertas que formen parte de las políticas activas del mercado de trabajo del Estado miembro de que se trate. Tanto los Estados de la Unión, como los miembros y socios de la red EURES, deben operar a través de una plataforma informática común, deben velar por su transparencia y visibilidad, deben poner en marcha los servicios de apoyo que sean necesarios para trabajadores y empresarios, y deben proporcionar a los usuarios información y orientación sobre oportunidades de empleo. También deben prestar, previa solicitud de trabajadores o empresarios, asistencia en el estadio posterior a la contratación. Todas las actuaciones deben llevarse a cabo, en fin, con arreglo a las normas europeas de protección de datos personales. La Comisión Europea estará asistida en esta materia por el Comité EURES, constituido por representantes de los Estados miembros conforme a los criterios establecidos, bien es cierto que con una proyección más general, por el Reglamento UE 182/2011 (que establece reglas y pautas básicas para canalizar el control por parte de los Estados miembros del ejercicio de las competencias de ejecución por parte de la Comisión).

\section{LAS MEDIDAS DE SIMPLIFICACIÓN DOCUMENTAL PARA FACILITAR LA LIBRE CIRCULACIÓN DE PERSONAS}

Desde el punto de vista personal, el Reglamento UE 2016/1191, de 6 de julio, tiene un radio de acción más amplio que la anterior norma 
comunitaria, pues se refiere, en general, a la libre circulación de ciudadanos de la Unión. Con todo, se trata también de una norma instrumental, en el sentido de que su regulación se dirige esencialmente a facilitar el cumplimiento de los trámites que implica un cambio de país de residencia para quienes ejercitan ese derecho. Como es regla general en este tipo de normas, y como enfatiza su preámbulo, el Reglamento UE 2016/1191 se inscribe en el objetivo comunitario de creación, mantenimiento y desarrollo de «un espacio de libertad, seguridad y justicia, sin fronteras interiores, en el que esté garantizada la libre circulación de personas», al que esta norma pretende contribuir mediante la simplificación de «los requisitos administrativos existentes para la presentación en un Estado miembro de determinados documentos públicos expedidos por las autoridades de otro Estado miembro». Para llevar adelante este propósito, el Reglamento 2016/1191 parte de dos presupuestos, de distinta naturaleza pero ambos muy pertinentes en este contexto: por un lado, del hecho de que los Estados miembros de la Unión son a su vez partes contratantes del Convenio de la Haya de 5 de octubre de 1961 («Convenio sobre la Apostilla»), por el que se suprime la exigencia de legalización de documentos públicos extranjeros y se simplifica la circulación de documentos públicos expedidos por las partes contratantes; por otro lado, del principio de confianza mutua que debe regir entre los socios comunitarios, también en este terreno. El Reglamento 2016/1191, por lo demás, deja vigentes los sistemas particulares que para estos fines de convalidación pudieran ser aplicables entre los Estados miembros, permite que entre ellos se siga utilizando el citado Convenio de la Haya, y mantiene el espacio de juego de otras normas de la Unión Europea que se ocupan, desde otras perspectivas, de la legalización de documentos o trámites similares, particularmente de las que tratan de la firma e identificación electrónicas.

Funcionalmente, el Reglamento 2016/1191 se aplica «a los documentos públicos expedidos por las autoridades de un Estado miembro de conformidad con su Derecho nacional que han de ser presentados a las autoridades de otro Estado miembro», siempre que dichos documentos se refieran a determinados hechos, acontecimientos o datos personales (separada o conjuntamente). Se aplica, concretamente, a documentos o trámites similares referidos a nacimiento, fe de vida, defunción, nombre, matrimonio (incluida capacidad para contraerlo, estado civil, divorcio, separación judicial o nulidad del matrimonio), unión de hecho registrada (así como cancelación de registro de la unión de hecho, separación judicial o anulación de dicha unión), filiación, adopción, domicilio o residencia, nacionalidad, 
ausencia de antecedentes penales y, en su caso, certificaciones que puedan exigirse para ejercer el derecho de sufragio activo y pasivo en un país distinto de la nacionalidad. Por propia disposición expresa, no se aplica el Reglamento 2016/1191, por el contrario, a los documentos expedidos por países terceros, ni a las copias certificadas de los documentos sobre nacimiento, ni «al reconocimiento en un Estado miembro de los efectos jurídicos relativos al contenido de los documentos públicos expedidos por las autoridades de otro Estado miembro». Se entiende por «documento público» a todos estos efectos el que dimana de una autoridad o funcionario vinculado a órganos jurisdiccionales de un Estado miembro, los documentos de carácter administrativo, las actas notariales, las certificaciones oficiales puestas sobre documentos privados, y los documentos expedidos por agentes diplomáticos o consulares. Se entiende por «autoridad» la autoridad pública de un Estado miembro o la entidad de carácter oficial autorizada por el Derecho nacional para expedir o recibir un documento público; se entiende por «legalización» el trámite de certificación de la autenticidad de la firma de un funcionario o cargo público, la calidad en la que ha actuado el signatario del documento y, en su caso, la identidad del sello o timbre que figure en el documento; se entiende por «trámite similar» la fijación de la apostilla contemplada en el Convenio de la Haya, y se entiende por «otros trámites» el requisito de presentación de copias certificadas y traducciones de documentos públicos.

La principal regla del Reglamento 2016/1191, y su principal aportación, es sin duda la declaración de exención de «toda forma de legalización y trámite similar» para los documentos que figuran en su campo de aplicación, así como para sus copias certificadas. Una regla complementaria también relevante es la simplificación de «otros trámites» relativos a las copias certificadas, de modo que cuando un Estado miembro exija la presentación del original de un documento público expedido por otro Estado miembro, no podrá exigir también copia certificada de dicho documento, con la precisión añadida de que cuando un Estado miembro permita la presentación de copia certificada, debe aceptar la copia certificada realizada en otro Estado miembro. Una segunda regla complementaria tiene por objeto la simplificación de «otros trámites» relativos a traducciones, de modo que no será exigible la traducción ni cuando el documento esté redactado en la lengua oficial del Estado miembro en el que se presente (o en la lengua oficial del lugar de presentación, si el Estado tuviera varias lenguas oficiales), ni, en los casos expresamente previstos a tal efecto (nacimiento, fe de vida y defunción, junto a algunos otros), cuando el documen- 
to vaya acompañado de «un impreso estándar multilingüe» en los términos previstos en el propio Reglamento (siempre que la autoridad a la que se le presente considere que la información incluida en dicho impreso es suficiente para la tramitación del documento).

Como es habitual en este tipo de normas, el Reglamento 2016/1191 también prevé algunos mecanismos de aplicación y supervisión. Con carácter general, dispone que para el pertinente intercambio de información entre los Estados miembros será utilizado el Sistema de Información del Mercado Interior (conocido como «IMI») establecido y regulado por el Reglamento UE 1024/2012, con la precisión de que las operaciones de intercambio y transmisión de información o de documentación entre los Estados miembros en virtud de lo dispuesto en este Reglamento «perseguirán la única finalidad de que las autoridades competentes comprueben la autenticidad de documentos públicos mediante el IMI». Declara el Reglamento 2016/589, en particular, que si las autoridades de un Estado miembro en el que se presente el documento o su copia certificada albergaran dudas razonables sobre su autenticidad (autenticidad de la firma, calidad en que hubiera actuado el signatario, identidad del sello o timbre, falsificación o alteración del documento), deberán formular solicitud de información a través de dicho sistema, y que los Estados miembros deben proceder a la designación de «al menos» una «autoridad central» para encargarse especialmente de atender estas solicitudes. El Reglamento 2016/1191, en fin, deja a salvo «las disposiciones legales, reglamentarias y administrativas de los Estados miembros en materia de acceso público a los documentos públicos». Entra en vigor a los veinte días de su publicación oficial (DOUE de 26 de julio de 2016), pero fija como inicio de su periodo de aplicación la fecha de 16 de febrero de 2019, excepto en algunas de sus previsiones, que se adelantan a diferentes fechas de 2017 o 2018. 